\title{
ON GALILEAN CONNECTIONS AND THE FIRST JET BUNDLE
}

\author{
JAMES D.E. GRANT AND BRAD LACKEY
}

\begin{abstract}
We express the first jet bundle of curves in Euclidean space as homogeneous spaces associated to a Galilean-type group. Certain Cartan connections on a manifold with values in the Lie algebra of the Galilean group are characterized as geometries associated to systems of second order ordinary differential equations. We show these Cartan connections admit a form of normal coordinates, and that in these normal coordinates the geodesic equations of the connection are second order ordinary differential equations. We then classify such connections by some of their torsions, extending a classical theorem of Chern involving the geometry associated to a system of second order differential equations.
\end{abstract}

\section{A preparatory: the Euclidean group and Riemannian geometry}

Recall that the Euclidean group of motions can be described as a matrix group:

$$
\operatorname{Eucl}_{n}=\left\{\left(\begin{array}{ll}
1 & 0 \\
x & A
\end{array}\right): x \in \mathbb{R}^{n}, A \in O_{n}\right\} .
$$

The canonical left-invariant Maurer-Cartan form for this group is

$$
\left(\begin{array}{cc}
1 & 0 \\
-A^{-1} x & A^{-1}
\end{array}\right)\left(\begin{array}{cc}
0 & 0 \\
d x & d A
\end{array}\right)=\left(\begin{array}{cc}
0 & 0 \\
A^{-1} d x & A^{-1} d A
\end{array}\right) \text {. }
$$

Writing this as $\left(\begin{array}{cc}0 & 0 \\ \omega^{i} & \omega^{i}{ }_{j}\end{array}\right)$, one can easily see the Maurer-Cartan equations for the component forms are the traditional structural equations of Euclidean geometry:

$$
\begin{aligned}
d \omega^{i}+\omega^{i}{ }_{j} \wedge \omega^{j} & =0 \\
d \omega^{i}{ }_{j}+\omega^{i}{ }_{k} \wedge \omega^{k}{ }_{j} & =0 .
\end{aligned}
$$

Euclidean space is represented as a homogeneous space associated to the Euclidean group, $\pi$ :

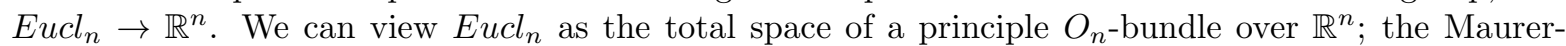
Cartan form is a $\mathfrak{e u c l}_{n}$-valued one form on the total space of the bundle which satisfies the Maurer-Cartan equations.

A Riemannian geometry is a manifold, $M$, and an ( $O_{n}$-valued) orthonormal frame bundle, $\pi: P \rightarrow$ $M$, together with $\mathfrak{e u c l}_{n}$-valued one form on the total space of the bundle, generically called a Cartan connection, but in this case traditionally termed a metrical or Riemannian connection. However, we do not require (and it may not be possible) that this matrix valued one-form satisfy the Maurer-Cartan equations. This failure is measured as torsion/curvature of the forms:

$$
d\left(\begin{array}{cc}
0 & 0 \\
\omega^{i} & \omega^{i}{ }_{j}
\end{array}\right)+\left(\begin{array}{cc}
0 & 0 \\
\omega^{i} & \omega_{k}^{i}
\end{array}\right) \wedge\left(\begin{array}{cc}
0 & 0 \\
\omega^{k} & \omega^{k}{ }_{j}
\end{array}\right)=\left(\begin{array}{cc}
0 & 0 \\
\Omega^{i} & \Omega^{i}{ }_{j}
\end{array}\right) .
$$

We may write

$$
\begin{aligned}
\Omega^{i} & =\frac{1}{2} T^{i}{ }_{j k} \omega^{j} \wedge \omega^{k} \\
\Omega^{i}{ }_{j} & =\frac{1}{2} R^{i}{ }_{j k l} \omega^{k} \wedge \omega^{l} .
\end{aligned}
$$

The tensors $T, R$ are the classical Riemannian torsion and curvature. The fundamental theorem of Riemannian geometry can be phrased as: the tensor $T$ parameterizes in a one-to-one fashion the metrical connections on $P$. In particular, there exists a unique connection such that $T=0$.

Date: 24 September 1999.

1991 Mathematics Subject Classification. 53C15, 58A20, $70 \mathrm{G} 35$.

Key words and phrases. Galilean group, Cartan connections, jet bundles, 2nd order ode's, KCC-theory.

This work was funded by the EPSRC, and the University of Hull. 


\section{Klein and Cartan geometries}

The essence of Klein's Erlangen programme was to realize the various forms of geometry that had arisen in the nineteenth century as homogeneous spaces associated to Lie groups. Specifically, these geometries have transitive automorphism groups, $G$; so if $H$ represents the isotropy group of a typical point, the original space is diffeomorphic to $G / H$. We have the canonical principle $H$-bundle $\pi: G \rightarrow$ $G / H$; the Maurer-Cartan forms of $G, \omega_{G}$, is a $\mathfrak{g}$-valued one-form which satisfies the Maurer-Cartan equations: $d \omega_{G}+\frac{1}{2}\left[\omega_{G}, \omega_{G}\right]=0$.

Cartan extended Klein's programme to include curved versions of geometries. In modern language, we consider an $H$-principle bundle over a smooth manifold, $\pi: P \rightarrow M$, and a $\mathfrak{g}$-valued one-form, $\omega \in \Omega^{1}(P, \mathfrak{g})$. This one-form, called the Cartan connection, is to satisfy:

1. for each $p \in P,\left.\omega\right|_{p}: T P \rightarrow \mathfrak{g}$ is an isomorphism;

2. for any $h \in H, R_{h}^{*} \omega=\operatorname{ad}\left(h^{-1}\right) \omega ;$ and,

3. for each $X \in \mathfrak{h}, \omega\left(X^{\dagger}\right)=X$.

These three conditions guarantee that at each $x \in M, T_{x} M \cong \mathfrak{g} / \mathfrak{h}$. Since the Maurer-Cartan equations are generally not satisfied, $M$ can be viewed as a curved version of $G / H$.

Fix $\pi: P \rightarrow M$ and a Cartan connection $\omega$. For any curve $\rho:[0,1] \rightarrow P$, the equation $\rho^{*} \omega=\tilde{\rho}^{*} \omega_{G}$ is a first order ordinary differential equation for the curve $\tilde{\rho}$ into $G$. Specifying an initial condition, say $\tilde{\rho}(0)=e$, and using the compactness of $[0,1]$, one gets a unique curve $\tilde{\rho}:[0,1] \rightarrow G$ called the development of $\rho$. Now, given a curve $\sigma:[0,1] \rightarrow M$, let $\rho$ be any lift of $\sigma$ to $P$. One can show that the development, $\tilde{\rho}$, depends upon the lift, but the projection of this curve to $G / H$ does not. Therefore, one gets a unique curve $\tilde{\sigma}:[0,1] \rightarrow G / H$ associated to $\sigma$, also called its development. The purpose of this exercise is that if one has a natural notion of 'straight line' in the model space $G / H$, then one can define geodesics in $M$ by taking curves whose developments are straight lines. This is precisely the way geodesics arise in Riemannian and projective geometry. See [5, $\$ \S \mathbf{3 . 7}$ and 5.4] for a more detailed treatment of developments of curves.

\section{The FIRST JET BUNDLE}

Our goal is to realize the first jet bundle $J^{1}\left(\mathbb{R}, \mathbb{R}^{n}\right)$ as a homogeneous space associated to its natural automorphism group. To this end, we introduce some features of the first jet bundle.

Formally, the first jet bundle, $J^{1}\left(\mathbb{R}, \mathbb{R}^{n}\right)$, is a vector bundle over $\mathbb{R} \times \mathbb{R}^{n}$ defined via equivalence classes of germs of curves from $\mathbb{R}$ into $\mathbb{R}^{n}$. Although it is much easier to understand the structure of the first jet bundle by using local coordinates, for completeness we present the formal definition as this is simple to generalize.

Definition 3.1. Fix $(t, x) \in \mathbb{R} \times \mathbb{R}^{n}$ and consider two germs of curves $\gamma_{1}, \gamma_{2}:(t-\epsilon, t+\epsilon) \rightarrow \mathbb{R}^{n}$ with $\gamma_{1}(t)=x=\gamma_{2}(t)$. We say these curves have first order contact at $(t, x)$ if they have the same tangent at $(t, x): \dot{\gamma}_{1}(t)=\dot{\gamma}_{2}(t)$. First order contact is clearly an equivalence relation, so denote $[\gamma]_{(t, x)}$ as the equivalence class of $\gamma$. The first jet bundle $J^{1}\left(\mathbb{R}, \mathbb{R}^{n}\right)$ is the vector bundle over $\mathbb{R} \times \mathbb{R}^{n}$ whose fibre over $(t, x)$ is $\left\{[\gamma]_{(t, x)} \mid \gamma:(t-\epsilon, t+\epsilon) \rightarrow \mathbb{R}^{n}, \gamma(t)=x\right\}$.

It is straight forward to justify that the definition above does indeed yield $J^{1}\left(\mathbb{R}, \mathbb{R}^{n}\right)$ as a vector bundle over $\mathbb{R} \times \mathbb{R}^{n}$. One way to see this is to identify $J^{1}\left(\mathbb{R}, \mathbb{R}^{n}\right) \cong \mathbb{R} \times T \mathbb{R}^{n}$. At each $(t, x)$, an equivalence class $[\gamma]_{(t, x)}$ determines a unique tangent vector to $\mathbb{R}^{n}$. Conversely, each tangent vector to $\mathbb{R}^{n}$ at $(t, x)$ can be realized as the tangent to a curve through $(t, x)$.

The identification $J^{1}\left(\mathbb{R}, \mathbb{R}^{n}\right) \cong \mathbb{R} \times T \mathbb{R}^{n}$ yields a preferred choice of coordinates. Specifically, if $\left(x^{j}\right)$ is a local coordinate system of $\mathbb{R}^{n}$, then tangent vectors are expressed as $y=y^{j} \frac{\partial}{\partial x^{j}}$. So we may always take local coordinates of the form $\left(t, x^{j}, y^{j}\right)$. Note that if we change coordinates, $\bar{x}=\bar{x}(x)$, we have both $d \bar{x}=A^{-1} d x$ and $\bar{y}=A^{-1} y$, where $A=\frac{\partial x}{\partial \bar{x}}$. Thus the 'contact' forms $d x-y d t$ have nice transformational character.

One cannot overestimate the utility of the contact forms. If we represent a curve in coordinates, $t \stackrel{\gamma}{\mapsto} x(t)$, then we can naturally define a curve on $J^{1}\left(\mathbb{R}, \mathbb{R}^{n}\right)$ by $t \mapsto\left(t, x(t), \frac{d x}{d t}(t)\right)$. This curve is called the first jet (or prolongation) of $\gamma$, and is traditionally denoted $j^{1} \gamma$. Now, if $\sigma: \mathbb{R} \rightarrow J^{1}\left(\mathbb{R}, \mathbb{R}^{n}\right)$, then $\sigma=j^{1} \gamma$ for some $\gamma$ if and only if $\sigma$ is integral to the contact forms: $\sigma^{*}(d x-y d t)=0$. Thus the contact forms completely encode the fact that $y$ is the derivative of $x$ with respect to $t$.

The first jet bundle, like Euclidean space or projective space, carries a canonical notion of straight line. Intuitively, a straight line is given by $x=a t$ and $y=a$ for some fixed $a \in \mathbb{R}^{n}$. Of course, if $a=0$, 
then the curve degenerates to just a point, and we wish to exclude this. We can express this picture using the contact forms together with $d t, d y$.

Definition 3.2. A curve $\tilde{\sigma}: \mathbb{R} \rightarrow J^{1}\left(\mathbb{R}, \mathbb{R}^{n}\right)$ is a straight line if $\tilde{\sigma}^{*}(d x-y d t)=\tilde{\sigma}^{*}(d y)=0$ and $\tilde{\sigma}^{*}(d t) \neq 0$.

\section{Galilean geometry}

Strictly speaking we are not considering the Galilean group; rather than taking the rotations, translations, and boosts on the tangent space, we take arbitrary affine transformations and boosts on the first jet space 1 Specifically, we define

$$
G a l_{n}=\left\{\left(\begin{array}{ccc}
1 & 0 & 0 \\
t & 1 & 0 \\
x & y & A
\end{array}\right): t \in \mathbb{R}, x, y \in \mathbb{R}^{n}, A \in G l_{n}\right\} .
$$

The canonical Maurer-Cartan forms for this group are

$$
\begin{gathered}
\left(\begin{array}{ccc}
1 & 0 & 0 \\
-t & 1 & 0 \\
-A^{-1}(x-y t) & -A^{-1} y & A^{-1}
\end{array}\right)\left(\begin{array}{ccc}
0 & 0 & 0 \\
d t & 0 & 0 \\
d x & d y & d A
\end{array}\right) \\
=\left(\begin{array}{ccc}
0 & 0 & 0 \\
d t & 0 & 0 \\
A^{-1}(d x-y d t) & A^{-1} d y & A^{-1} d A
\end{array}\right) .
\end{gathered}
$$

The isotropy group of a point in the first jet bundle is

$$
H=\left\{\left(\begin{array}{lll}
1 & 0 & 0 \\
0 & 1 & 0 \\
0 & 0 & A
\end{array}\right): A \in G l_{n}\right\} .
$$

Clearly $J^{1}\left(\mathbb{R}, \mathbb{R}^{n}\right) \cong G a l_{n} / H$.

Definition 4.1. A Galilean manifold is a smooth manifold $X$, equipped with an principle $H$-bundle, $\pi: P \rightarrow X$, and a Cartan connection on $P$ with values in $\mathfrak{g a l}_{n}$.

To fix our notation, we will write the Cartan connection of our Galilean manifold as $\left(\begin{array}{ccc}0 & 0 & 0 \\ \tau & 0 & 0 \\ \omega & \phi & \Pi\end{array}\right)$. The curvature of our connection is then

$$
\left(\begin{array}{ccc}
0 & 0 & 0 \\
T & 0 & 0 \\
\Omega & \Phi & R
\end{array}\right)=\left(\begin{array}{ccc}
0 & 0 & 0 \\
d \tau & 0 & 0 \\
d \omega+\Pi \wedge \omega+\phi \wedge \tau & d \phi+\Pi \wedge \phi & d \Pi+\Pi \wedge \Pi
\end{array}\right) .
$$

Curvature measures the failure of the geometric structure to be representable in coordinate systems. In the first jet bundle, we may always take adapted coordinates of the form $(t, x, y)$; but for the most general Galilean geometries this is not possible even locally. Therefore, we are really interested in Cartan connections which are "partially integrable."

Definition 4.2. The Cartan connection of a Galilean manifold, $X$, is holonomic if $T=0$ and torsionfree if $\Omega=0$. If both conditions are satisfied, we will call $X$ a normal Galilean manifold.

We use the overworked term 'normal' for this type of Galilean manifold because it has normal coordinates in some sense. The following theorem is a precise statement of this; intuitively, we have demanded certain curvatures vanish in order to find local coordinates which mimic the adapted coordinates of the first jet bundle.

Theorem 4.3. Let $X$ be a normal Galilean manifold. Take any point in $X$. Then about this point, there exists a coordinate system, $(t, x, y)$, and a local section $h$ of $P$, such that $h^{*} \tau=d t, h^{*} \omega=d x-y d t$, and $h^{*} \phi=d y(\bmod d t, d x)$.

\footnotetext{
${ }^{1}$ In what follows, one can use the traditional Galilean group, but the resulting geometry is slightly different. It is, in fact, that proposed in [3].
} 
JAMES D.E. GRANT AND BRAD LACKEY

Proof. Let $\tilde{h}$ be any local section of $P$ near our given point. Immediately we have $d \tilde{h}^{*} \tau=0$, and hence restricting our domain if necessary $\tilde{h}^{*} \tau=d t$. As $d t \neq 0$, the function $t$ serves as a coordinate function.

Define $\mathcal{I}$ to be the exterior ideal generated by the one-forms $d t$ and $\tilde{h}^{*} \omega$. The curvature conditions $T=0$ and $\Omega=0$ show that $\mathcal{I}$ is integrable. Since $d t \in \mathcal{I}$ each integral manifold lies in a $t=$ constant hypersurface. Again restricting our domain if necessary, we may choose independent functions $x^{j}$ so that the integral manifolds of $\mathcal{I}$ are uniquely described by $t=$ constant and $x=$ constant.

Note that the integral manifolds of $\mathcal{I}$ are also the integral manifolds of $\left\langle d t, d x^{j}\right\rangle$. Therefore, at each point of our domain we may express $\tilde{h}^{*} \omega=A(d x-y d t)$ for some invertible matrix $A$ and vector $y$. We claim that $\left\{y^{j}\right\}$ serve as coordinates of the integral manifolds, and hence completes our local coordinate system. To show this, it suffices to show that $\{d t, d x, d y\}$ forms a local coframe near our given point. To this end, we compute:

$$
d \tilde{h}^{*} \omega=d A \cdot A^{-1} \wedge \tilde{h}^{*} \omega-A d y \wedge d t .
$$

Yet, as $\Omega=0$, we have the structure equation $d \omega=-\Pi \wedge \omega-\phi \wedge \tau$. Therefore,

$$
\left(d A \cdot A^{-1}+\tilde{h}^{*} \Pi\right) \wedge \tilde{h}^{*} \omega=\left(A d y-\tilde{h}^{*} \phi\right) \wedge d t .
$$

Every two-form in the left side of this equation contains an $\tilde{h}^{*} \omega$. Thus, $A d y-\tilde{h}^{*} \phi=N \tilde{h}^{*} \omega+\lambda d t$ for some matrix $N$ and vector $\lambda$. Yet, $\tau, \omega, \phi$ are linearly independent. Therefore, the $d y^{j}$ are linearly independent, and also independent from $\tilde{h}^{*} \omega, \tilde{h}^{*} \tau$, and thus independent from $d t, d x$ as well.

Finally, define a new section by $h=R_{A} \tilde{h}$, where $A$ abbreviates $\left(\begin{array}{ccc}1 & 0 & 0 \\ 0 & 1 & 0 \\ 0 & 0 & A\end{array}\right) \in H$. Then we have $h^{*} \tau=\tilde{h}^{*} \tau, h^{*} \omega=A^{-1} \tilde{h}^{*} \omega$, and $h^{*} \phi=A^{-1} \tilde{h}^{*} \phi$. In particular, $h^{*} \tau=d t, h^{*} \omega=d x-y d t$, and $h^{*} \phi=d y$ $\left(\bmod h^{*} \tau, h^{*} \omega\right)$, as desired.

Definition 4.4. A geodesic in a Galilean manifold is a (germ of a) curve $\sigma:(-a, a) \rightarrow X$ whose development $\tilde{\sigma}:(-a, a) \rightarrow J^{1}\left(\mathbb{R}, \mathbb{R}^{n}\right)$ is contained in a straight line.

Proposition 4.5. Let $X$ be a normal Galilean manifold, and let $(t, x, y)$ be normal coordinates in $X$, and write $h^{*} \phi=d y+\Gamma(t, x, y) d t+N(t, x, y)(d x-y d t)$. Then the geodesics of $X$ are the solutions to the system of second order differential equations

$$
\frac{d^{2} x^{j}}{d t^{2}}+\Gamma^{j}\left(t, x, \frac{d x}{d t}\right)=0 .
$$

Proof. We have already seen that the straight lines in $J^{1}\left(\mathbb{R}, \mathbb{R}^{n}\right)$ are characterized by the equations $\tilde{\sigma}^{*}(d x-y d t)=0$ and $\tilde{\sigma}^{*}(d y)=0$. Yet, $d x-y d t$ and $d y$ are the $\omega$ and $\phi$ terms, respectively, in the Maurer-Cartan forms of the Galilean group. Thus by the definition of development, a curve $\sigma$ is a geodesic if and only if it is non-degenerate and $\sigma^{*}\left(h^{*} \omega\right)=\sigma^{*}\left(h^{*} \phi\right)=0$. Yet, in normal coordinates $h^{*} \omega=d x-y d t$ and $h^{*} \phi=d y+\Gamma d t+N(d x-y d t)$; the integral curves to these forms are precisely the solutions to (11).

Theorem 4.6. Consider local coordinates $(t, x, y)$, and curves in this coordinate system given by (因). Then we have the following

(i) There are many normal Galilean geometries having these coordinates as normal coordinates and (1) as geodesic equations. For these we have

$$
\Phi^{i}=D^{i}{ }_{j} \tau \wedge \phi^{j}+Q^{i}{ }_{j k} \omega^{j} \wedge \phi^{k}+P^{i}{ }_{j} \tau \wedge \omega^{j}+\frac{1}{2} T^{i}{ }_{j k} \omega^{j} \wedge \omega^{k} .
$$

(ii) For the geometries described in (i), the tensor D parameterizes in a one-to-one fashion the forms $\phi$. There are many such normal Galilean geometries for each choice of $D$.

(iii) To each geometry described in (ii), the symmetric part of the tensor $Q, Q^{i}{ }_{(j k)}=\frac{1}{2}\left(Q^{i}{ }_{j k}+Q^{i}{ }_{k j}\right)$, parameterizes in a one-to-one fashion the forms $\Pi$. There is precisely one such normal Galilean geometry for each choice of $Q^{i}{ }_{(j k)}$.

Proof. Let $(t, x, y)$ be as given; on the portion of $P$ over this coordinate system we have natural coordinates $(t, x, y, A)$. From the construction of the coordinate $t$, it is clear that $\tau=d t$. Similarly, if there is a section $h$ so that $h^{*} \omega=d x-y d t$, then we must have $\omega=A^{-1}(d x-y d t)$ (explicitly, the desired section is 
then $h(t, x, y)=(t, x, y, A(t, x, y)))$. This implies that $\phi=A^{-1} d y(\bmod \tau, \omega)$. The geodesics of the geometry are given by $\sigma^{*} \omega=\sigma^{*} \phi=0$, hence $\phi=A^{-1}(d y+\Gamma d t)(\bmod \omega)$, say $\phi=A^{-1}(d y+\Gamma d t+N(d x-y d t))$ where $N$ is an arbitrary matrix. If the structure equation $d \omega=-\Pi \wedge \omega-\phi \wedge \tau$ is to be satisfied, we must have $\Pi^{i}{ }_{j}=\left(A^{-1} d A\right)^{i}{ }_{j}+\left(A^{-1}\right)^{i}{ }_{k} N^{k}{ }_{l} A^{l}{ }_{j} d t+\left(A^{-1}\right)^{i}{ }_{l} \Gamma_{r s}^{l} A^{r}{ }_{j} A^{s}{ }_{k} \omega^{k}$, where $\Gamma^{i}{ }_{j k}=\Gamma^{i}{ }_{k j}$ but is otherwise arbitrary. Hence (i) is shown. Moreover, the most general Galilean geometry satisfying (i) is

$$
\begin{aligned}
\tau & =d t \\
\omega^{i} & =\left(A^{-1}\right)^{i}{ }_{j}\left(d x^{j}-y^{j} d t\right) \\
\phi^{i} & =\left(A^{-1}\right)^{i}{ }_{j}\left(d y^{j}+\Gamma^{j} d t+N^{j}{ }_{k}\left(d x^{k}-y^{k} d t\right)\right) \\
\Pi^{i}{ }_{j} & =\left(A^{-1} d A\right)^{i}{ }_{j}+\left(A^{-1}\right)^{i}{ }_{k} N^{k}{ }_{l} A^{l}{ }_{j} d t+\left(A^{-1}\right)^{i}{ }_{l} \Gamma^{l}{ }_{r s} A^{r}{ }_{j} A^{s}{ }_{k} \omega^{k} .
\end{aligned}
$$

We leave it as an easy exercise to the reader to show that for these forms, $\Phi$ has no $\phi \wedge \phi$ term.

To show (ii), we compute

$$
\begin{aligned}
\Phi^{i} & =d \phi+\Pi \wedge \phi \\
& =\left(A^{-1}\right)^{i}{ }_{j}\left(2 N^{j}{ }_{k}-\frac{\partial \Gamma^{j}}{\partial y^{k}}\right) A^{k}{ }_{l} \tau \wedge \phi^{l} \quad(\bmod \omega) .
\end{aligned}
$$

Therefore, for any choice of $D$, we have $N=\frac{1}{2}\left(\frac{\partial \Gamma}{\partial y}+A D A^{-1}\right)$.

Finally, suppose $D$, and hence $N$, is now fixed. Note that we may write $\phi=A^{-1}\left(d y+\Gamma d t+\frac{1}{2} \frac{\partial \Gamma}{\partial y}(d x-y d t)\right)+D \omega$. One easily computes the $\omega^{j} \wedge \phi^{k}$ term in $\Phi^{i}$ to be

$$
\left(A^{-1}\right)^{i}{ }_{l}\left(\Gamma_{r s}^{l}-\frac{1}{2} \frac{\partial^{2} \Gamma^{l}}{\partial y^{r} \partial y^{s}}-\frac{1}{2} A_{m}^{l} \frac{\partial D^{m}{ }_{p}}{\partial y^{s}}\left(A^{-1}\right)^{p}{ }_{r}\right) A^{r}{ }_{j} A^{s}{ }_{k} \omega^{j} \wedge \phi^{k} .
$$

Only the symmetric part of this involves $\Gamma_{j k}^{i}$, hence specifying $Q^{i}{ }_{(j k)}$ determines $\Gamma_{j k}^{i}$ uniquely, and (iii) is proven.

This theorem generalizes the classical theorem of Chern about the geometry induced by systems of second order ordinary differential equation. We can state his result in our language as the following.

Corollary 4.7 (Chern [1]). To each system of second order ordinary differential equations, (1), there exists a unique normal Galilean connection such that $\Phi=0(\bmod \omega)$.

Proof. Choosing $D^{i}{ }_{j}=0$ and $Q^{i}{ }_{(j k)}=0$, we see that $\Phi=0(\bmod \omega)$.

\section{Appendix A. Geometry of Jet Bundles}

Jet spaces in generality have a great deal of structure. In recent years, it has become popular to study the geometry of jet bundles, see for instance [4]; however, the usual techniques involve nonlinear connections in the tangent bundle of the jet bundle. Throughout the paper we have used the exterior differential calculus, as this formalism is very convenient for studying Cartan geometries. In this appendix, we indicate how the results of the paper impact upon the geometry of jet spaces.

Let $B$ be a smooth manifold, and suppose $\pi: X \rightarrow B$ is a fibre bundle with fibre $F$. The tangent vectors to the fibres have good transformational character, so form a vector subbundle of $T X$ called the vertical vector space, and denoted as $V T X$. The easy way to see this is to note that $\xi$ is tangent to the fibre if and only if $\pi_{*} \xi=0$, hence $V T X=\operatorname{ker} \pi_{*}$. Unfortunately, although vectors tangent to $B$ locally complement the vertical vector space, they do not transform properly on $X$. Thus $T B$ is not a subbundle of $T X$. A nonlinear connection is merely a selection of complementary vector bundle to $V T X$ in $T X$. This bundle is usually called the horizontal vector space, and denoted $H T X$.

Another definition of a nonlinear connection is via the short exact sequence of vector bundles over $X$ :

$$
0 \rightarrow V T X \rightarrow T X \stackrel{\pi_{*}}{\rightarrow} \pi^{*} T B \rightarrow 0,
$$

where $\pi^{*} T B$ is the pull-back of the tangent of $B$ over $X$. A nonlinear connection is a splitting of this short exact sequence: $T X \cong V T X \oplus H T X$ where $H T X \cong \pi^{*} T B$. Unfortunately, without further knowledge about the structure of $X$, there is no canonical splitting.

Now we specialize to the case where $B=\mathbb{R} \times M$ for some smooth manifold $M$, and $X=J^{1}(\mathbb{R}, M)$. We wish to describe a nonlinear connection in local adapted coordinates $(t, x, y)$. The vertical vector 
space over this coordinate chart is spanned by $\left\{\frac{\partial}{\partial y^{j}}\right\}$, and $\pi^{*} T(\mathbb{R} \times M)$ is spanned by $\left\{\frac{\partial}{\partial t}+y^{j} \frac{\partial}{\partial x^{j}}, \frac{\partial}{\partial x^{j}}\right\}$. Do note that our choice of vector fields in the latter contains the contact structure of $J^{1}(\mathbb{R}, M)$.

Over our coordinate system, a selection of nonlinear connection is just a selection of functions $\Gamma, N$ such that

$$
H T J^{1}(\mathbb{R}, M)=\operatorname{span}\left\{\frac{\partial}{\partial t}+y^{j} \frac{\partial}{\partial x^{j}}-\Gamma^{j}(t, x, y) \frac{\partial}{\partial y^{j}}, \frac{\partial}{\partial x^{j}}-N_{k}^{j}(t, x, y) \frac{\partial}{\partial y^{k}}\right\} .
$$

For convenience, let us adopt the notation $\frac{d}{d t}=\frac{\partial}{\partial t}+y^{j} \frac{\partial}{\partial x^{j}}-\Gamma^{j}(t, x, y) \frac{\partial}{\partial y^{j}}$ and $\frac{\delta}{\delta x^{j}}=\frac{\partial}{\partial x^{j}}-N^{j}{ }_{k}(t, x, y) \frac{\partial}{\partial y^{k}}$.

We see that the nonlinear connection is expressed as two sets of function. The functions $\Gamma$ describe a second order ordinary differential equation, and as we have seen, the geometry of the first jet bundle is closely tied to these differential equations. One goal of studying this type of geometry is to determine a natural choice of the functions $N$ based upon a selection of $\Gamma$. The most natural thing to do is compute the commutators of the vector fields above. Oddly enough, this is not the proper thing to do.

Instead, it is far better to study affine connections on $T J^{1}(\mathbb{R}, M)$. We are not interested in arbitrary affine connections, but rather ones which reflect the structure of the first jet space. In other words we are not interested in Cartan connections with values in $\mathfrak{a f f}_{2 n+1}$ but rather those with values in $\mathfrak{g a l}_{n}$. That is, a normal Galilean connections yields a covariant derivative with structure:

$$
\begin{aligned}
& \nabla_{\frac{d}{d t} \frac{d}{d t}=0}=\nabla_{\frac{\delta}{\delta x^{k}} \frac{d}{d t}}=0 \quad \nabla_{\frac{\partial}{\partial y^{k}}} \frac{d}{d t}=0 \\
& \nabla_{\frac{d}{d t}} \frac{\delta}{\delta x^{k}}=N_{k}^{j} \frac{\delta}{\delta x^{j}} \quad \nabla_{\frac{\delta}{\delta x^{k}}} \frac{\delta}{\delta x^{k}}=\Gamma_{j k}^{l} \frac{\delta}{\delta x^{l}} \quad \nabla_{\frac{\partial}{\partial y^{k}}} \frac{\delta}{\delta x^{k}}=0 \\
& \nabla_{\frac{d}{d t}} \frac{\partial}{\partial y^{k}}=N_{k}^{j} \frac{\partial}{\partial y^{j}} \quad \nabla_{\frac{\delta}{\delta x^{k}}} \frac{\partial}{\partial y^{k}}=0 \quad \nabla_{\frac{\partial}{\partial y^{k}}} \frac{\partial}{\partial y^{k}}=\Gamma_{j k}^{l} \frac{\partial}{\partial y^{k}} .
\end{aligned}
$$

Now, it is a simple matter to see that the components of torsion determine the nonlinear and affine connection coefficients. For instance,

$$
\nabla_{\frac{\partial}{\partial y^{k}}} \frac{d}{d t}-\nabla_{\frac{d}{d t}} \frac{\partial}{\partial y^{k}}-\left[\frac{\partial}{\partial y^{k}}, \frac{d}{d t}\right]=-\frac{\delta}{\delta x^{k}}+\left(\frac{\partial \Gamma^{j}}{\partial y^{k}}-2 N_{k}^{j}\right) \frac{\partial}{\partial y^{j}} .
$$

\section{REFERENCES}

[1] S.S. Chern, Sur la géometrie d'un système d'equations différentialles du second ordre, Bull. Sci. Math. 63(1939), 206-212.

[2] B. Lackey, On adaptive control systems with application in biology, Open Systems and Information Dynamics, to appear.

[3] B. Lackey, Metric equivalence of path spaces, Nonlinear Analysis B, to appear.

[4] D.J. Saunders, The Geometry of Jet Bundles, Cambridge University Press, 1989.

[5] R.W. Sharpe, Differential Geometry, Springer-Verlag, 1998.

Department of Mathematics, University of Hull, Hull Hu6 7RX, United Kingdom

E-mail address: J.D.Grant@maths.hull.ac.uk

E-mail address: B.Lackey@maths.hull.ac.uk 